\title{
GAN MEMS RESONATOR USING A FOLDED PHONONIC CRYSTAL STRUCTURE
}

\author{
Siping Wang , Laura C. Popa, and Dana Weinstein \\ Department of Electrical Engineering and Computer Science \\ Massachusetts Institute of Technology, Cambridge, MA
}

\begin{abstract}
We present a Gallium Nitride (GaN) Lamb Wave resonator using a Phononic Crystal ( $\mathrm{PnC})$ to selectively confine elastic vibrations with wide-band spurious mode suppression. A unique feature of the design demonstrated here is a folded PnC structure to relax energy confinement in the non-resonant dimension and to enable routing access of piezoelectric transducers inside the resonant cavity. This provides a clean spectrum over a wide frequency range and improves series resistance relative to transmission line or tethered resonators by allowing a low-impedance path for drive and sense electrodes. GaN resonators are demonstrated with wide-band suppression of spurious modes, $f . Q$ product up to $3.06 \times 10^{12}$, and resonator coupling coefficient $k_{e f f}^{2}$ up to $0.23 \%$ (filter BW up to $0.46 \%$ ). Furthermore, these $\mathrm{PnC} \mathrm{GaN}$ resonators exhibit record-breaking power handling, with IIP3 of $+27.2 \mathrm{dBm}$ demonstrated at $993 \mathrm{MHz}$.
\end{abstract}

\section{INTRODUCTION}

High $Q$, small footprint MEMS resonators are very promising for building blocks in RF wireless communication, timing, inertial navigation, and sensing applications. Their potential for monolithic integration with circuits provides critical benefits such as the elimination of parasitic capacitance and inductance from bond pads and off-chip routing, size, weight, and power scaling, and simplification of fabrication and packaging. Recent advances in GaN Monolithic Microwave IC (MMIC) technology have made it an attractive platform for the realization of high performance MEMS resonators. With GaN's wide band gap (3.4 eV), high 2DEG mobility, and high piezoelectric coefficients, integration of GaN MEMS with High Electron Mobility Transistors (HEMTs) presents many opportunities for high power, high frequency applications.

This work focuses on the development of MEMS resonators for channel-select filtering in RF receiver front ends. For a MEMS band pass filter, the presence of spurious modes in the constituent resonators strongly impacts filter performance. Resonators with a clean frequency spectrum help reduce ripples in the pass-band and prevent interference from unwanted signals outside the pass-band. Conventional MEMS resonator designs with free mechanical boundaries are inherently prone to spurious modes, since free boundaries act as acoustic reflectors over all frequencies. To resolve this issue, the resonator boundary needs to be frequency selective.

One way to define the MEMS resonator cavity with frequency selective confinement is by using Phononic Crystals (PnCs), which involve periodic scatters to achieve highly reflective boundary conditions only for frequencies in a specific range. This acoustic band gap can be engineered based on the unit cell size and material configuration. Research in micro-scale PnCs has progressed rapidly in the past decade with band gap optimization at $\mathrm{GHz}$ frequencies in $\mathrm{Si}$ and $\mathrm{SiC}$ [1,2] and high- $Q$ resonators in $\mathrm{Si}, \mathrm{AlN}$ and $\mathrm{ZnO}[3,4,5]$. High- $Q$ resonant cavities using PnCs have been previously defined either as defect modes in a uniform 2D PnC (Fig. 1(a)) or as a suspended slab with free boundaries in the non-resonant dimension (Fig. 1(b)). While the acoustic band gap of these PnCs helps reduce resonance outside the band gap, these structures provide no spurious mode suppression inside the band gap. Furthermore, transducers must be routed through the $\mathrm{PnC}$ in these configurations, leading to resistive loading of $Q$. In this work, we demonstrate a new resonant structure leveraging both $\mathrm{PnC}$ acoustic confinement and the

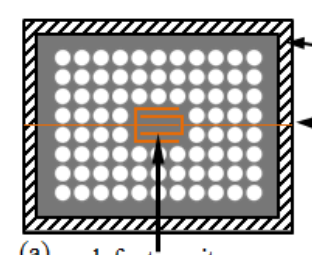

(a) defect cavity
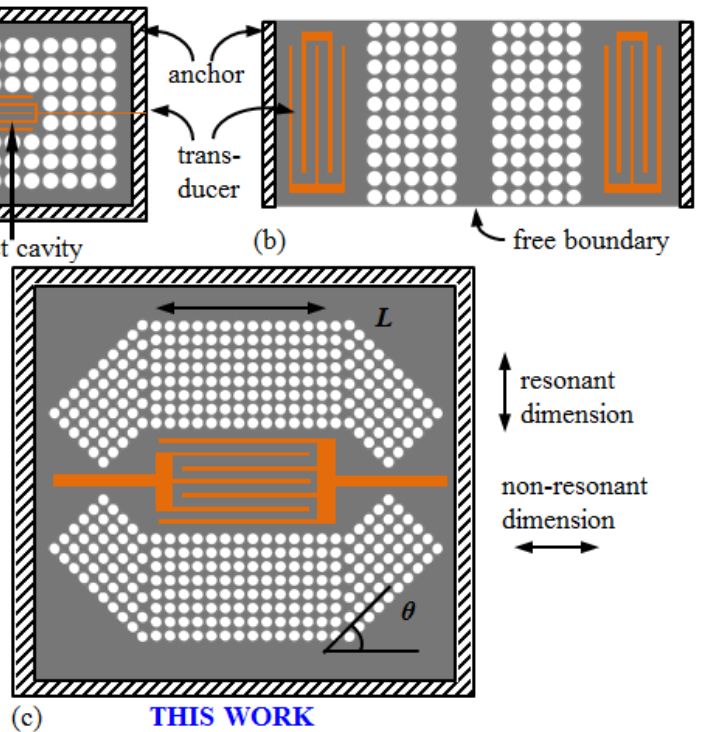

Figure 1: (a) Defect cavity in 2D PnC. Transducer routes through $P n C$ to cavity. (b) Transmission line PnC resonator, with transducers external to the resonance cavity (c) This work: Folded PnC resonator enabling transducer inside resonant cavity with spurious mode suppression, high-Q mode, and large power handling.

electromechanical benefits of GaN. The proposed GaN folded PnC structure (Fig. 1(c)) provides several important benefits:

- wide-band spurious mode suppression, both outside and inside the PnC band gap, through relaxed confinement in the non-resonant dimension,

- low-loss electrical routing to the resonant cavity to incorporate drive and sense transducers inside the resonator,

- improved heat dissipation relative to other PnC or tethered resonators, and

- robust design that is immune to residual stress and handling.

Using the folded PnC design, these improvements can be achieved while maintaining quality factor and transducer coupling comparable to traditional tethered resonators.

\section{DESIGN AND SIMULATION}

A square lattice $\mathrm{PnC}$ was chosen to define the resonant cavity of the folded $\mathrm{PnC}$ resonator. The PnC unit cell is a square block with a finite thickness defined by the GaN layer thickness, and a circular hole at the center as illustrated in Fig. 2(a). For the irreducible Brillouin zone (IBZ) in Fig. 2(b), the PnC band structure of the 537 $\mathrm{MHz}$ (unit cell $a=5.6 \mu \mathrm{m}$ ) resonators in this work is given in Fig. 2(c). It should be noted that the PnC does not have a complete band gap. Rather, there are only band gaps from $\mathrm{O}$ to $\mathrm{X}$ and from $\mathrm{X}$ to $\mathrm{M}$ but not from $\mathrm{O}$ to $\mathrm{M}$. This partial band gap is sufficient for the designed resonator since the $\mathrm{PnC}$ needs to be reflective only in the resonant dimension.

While the PnC resonator inherently suppresses spurious modes outside the band gap, it was found that the majority of spurious modes found inside the band gap are due to harmonics established in the non-resonant dimension. Energy confinement along the non-resonant dimension must therefore be relaxed while 
(a)

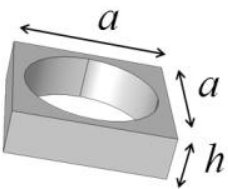

(b)

(c)

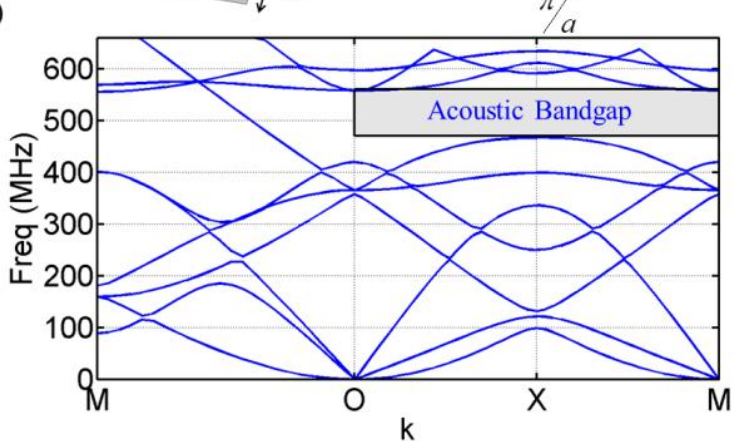

Figure 2: (a) Unit cell of the PnC structure. (b) Irreducible Brillouin zone in $k$ space. (c) PnC band structure, showing a partial band gap along edges of unit cell ( $O$ to $X$ to $M$ ).

maintaining high $Q$ for the fundamental mode. These design considerations motivated the folded-PnC structure, in which square lattice $\mathrm{PnC}$ segments are designed at a folding angle $\theta$ relative to the main $\mathrm{PnC}$ to provide good confinement for the desired mode while minimizing standing waves formed in the non-resonant dimension.

\section{A. Performance dependence on folding angle $\theta$}

Using 3D finite element method (FEM) simulations in COMSOL, folded $\mathrm{PnC}$ resonators with varying folding angle $\theta$ were investigated for their quality factor and spurious-free frequency range (SFFR). In these devices, $Q$ is limited by acoustic losses to the substrate. To capture this loss in the FEM simulation, Perfectly Matched Layers (PMLs) were implemented on the perimeter of the device beyond the PnC. Simulations included Multiphysics piezoelectric transduction to determine the 1-port response of the resonator. $Q$ and SFFR were then extracted from a multi-pole fit to the 1-port admittance of the resonator. Simulated results of the angular dependence of $Q$ and SFFR are shown in Fig. 3(a), where the angle $\theta=$ N/A corresponds to a PnC resonator of length $L$ with completely open boundaries in the non-resonant dimension (no folded PnC segments). The simulated $\mathrm{GaN}$ resonator with interdigitated transducer (IDT) has a $7^{\text {th }}$ harmonic $\mathrm{S}_{0}$ Lamb mode around $537 \mathrm{MHz}$, using a PnC unit cell length of $\mathrm{a}=5.6 \mu \mathrm{m}$. A constant folded segment length of 5 unit cells was chosen for this design. The PnC band gap is established between 467 and $562 \mathrm{MHz}$.

As can be seen in Fig. 3(a), the presence of the folded PnC improves $Q$ by a factor of over 2 above a critical angle between $30-45^{\circ}$, but tends to introduce spurious modes near the desired resonance. This can be explained by the role of the folded PnC segments in reflecting some radiated energy from the openings for all frequencies within the band gap, providing high $Q$ for the fundamental mode but enabling standing waves orthogonal to the resonance which result in additional modes within the band gap.

At all angles of the folded PnC segments, and even in the limiting case where no folded segments exist $(\theta=\mathrm{N} / \mathrm{A})$, undesired harmonic modes along the non-resonant dimension are established due to the finite length of the $\mathrm{PnC}$ and driving electrodes which results in a break in translational symmetry. Fig. 4 plots the 1-port frequency response of a simulated folded PnC resonator and the mode shapes of its main resonance and spurious modes. A device with longer cavity length $(L / a=28)$ is chosen for better illustration
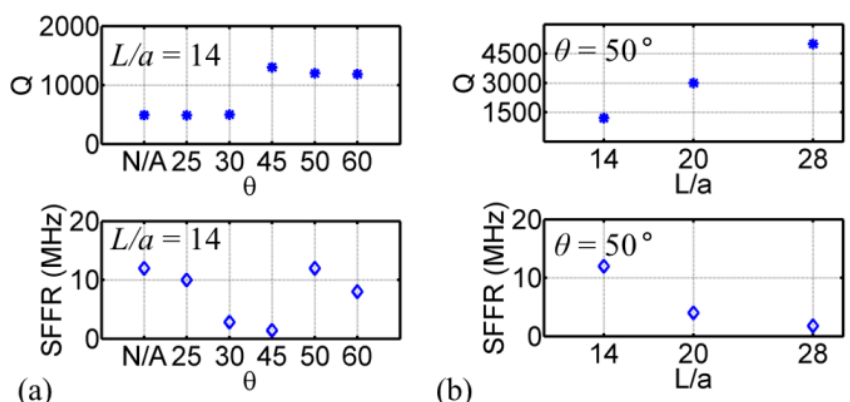

(b)

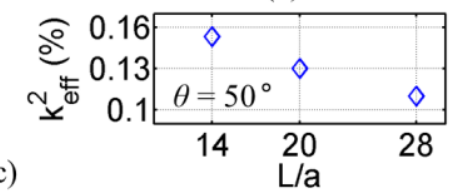

Figure 3: COMSOL simulated resonator performance, including $Q$, spurious-free frequency range (SFFR), and resonator coupling coefficient $k_{\text {eff }}^{2}$ for varying PnC folding angle $\theta$ and cavity length $L$, normalized to the PnC unit cell length ( $a=5.6 \mu \mathrm{m}$ at $537 \mathrm{MHz}$ ).

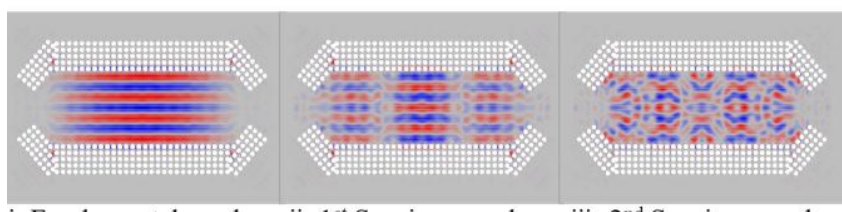

i. Fundamental mode ii. $1^{\text {st }}$ Spurious mode iii. $2^{\text {nd }}$ Spurious mode

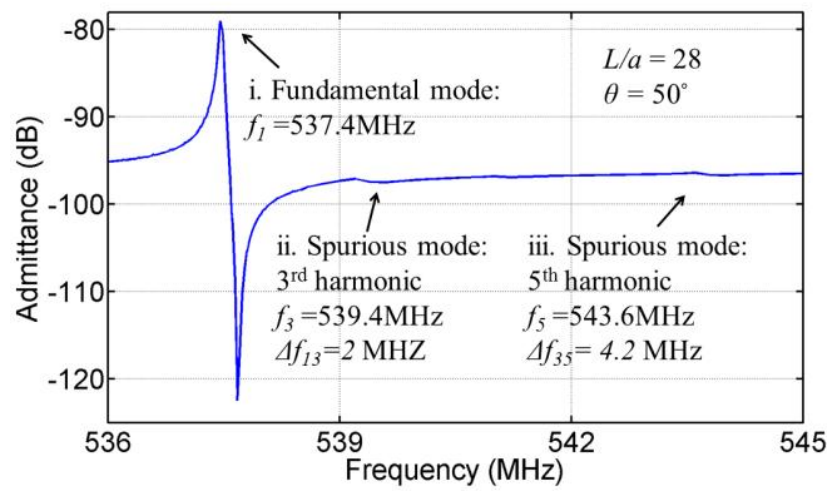

Figure 4: Simulated resonator mode shape and frequency spectrum. Compressive strain field along the resonant dimension is plotted.

of harmonics in the non-resonant dimension and the two spurious modes established within the band gap correspond to the $3^{\text {rd }}$ and $5^{\text {th }}$ harmonics. Even harmonics are suppressed due to symmetry of the driving and sensing electrodes. For a fundamental mode frequency $f_{l}$ centered in the band gap, the frequency of the $\mathrm{n}^{\text {th }}$ harmonic spurious mode can be approximated to first order by

$$
f_{n}=\sqrt{f_{0}^{2}+\left(\frac{n v}{L}\right)^{2}}
$$

where $v$ is the wave velocity along the non-resonant dimension and $f_{0}$ is the resonance frequency of the $\mathrm{S}_{0}$ Lamb mode in the case when cavity length $L$ is infinite. As can be seen in Fig. 4, these harmonics are significantly attenuated relative to the fundamental mode. This attenuation is attributed to signal cancellation in the IDT and to the openings designed on the central axis of the folded PnC structure, which not only route the transducer signal to the resonant cavity, but also relax energy confinement for waves traveling in the non-resonant dimension. 
At certain folding angles, additional anharmonic spurious modes not predicted by Eqn. 1 arise due to scattering off the "defect" at the folding point of the PnC. It is found from FEM simulation that at $\theta=50^{\circ}$, these spurious modes are entirely suppressed, achieving SFFR comparable to the case of no folded segments $(\theta=\mathrm{N} / \mathrm{A})$. Moreover, at $\theta=50^{\circ}$ resonator $Q$ is twice as high as that of the resonator without folded segments. Hence, this folding angle was selected for all designs in this work.

\section{B. $\quad$ Performance dependence on cavity length $L$}

A similar trade-off exists between $Q$ and SFFR as a function of cavity length $L$, which also strongly affects resonator $k_{\text {eff }}^{2}$. Simulation results of this dependence are presented in Fig. 3(b) and (c). As $L$ increases, resonator volume and therefore total stored energy increase. Meanwhile, energy loss is dominated by radiative losses at the PnC openings on the resonator's central axis. For a given folding angle and folded $\mathrm{PnC}$ aperture at the openings, the ratio between stored energy and energy loss increases with $L$ leading to a higher $Q$. A larger $L$ also leads to smaller SFFR since increasing $L$ brings spurious modes closer to the main peak, consistent with Eqn. 1. On the other hand, shorter resonators will have spurious modes pushed to higher frequencies, causing those modes to be more attenuated as they are closer to the PnC band edge. For a short enough cavity, spurious modes can even be pushed outside the PnC band gap and become significantly attenuated, potentially eliminating all spurious modes inside the band gap. However, such design requires very small $L$, resulting in a reduced $Q$ of the fundamental mode and increased motional impedance.

The decrease in $k_{\text {eff }}^{2}$ with increasing $L$ (Fig. 3(c)) is mainly a side-effect of the increase in $Q$. For the same IDT transducer configuration, scaling the cavity length $L$ does not affect $C_{m} / C_{o}$, where $C_{m}$ is the motional capacitance of the resonator and $C_{o}$ is the nominal capacitance of the transducer, since both the mode and electrodes extend equally with $L$ in the non-resonant dimension. When $Q \sim C_{d} / C_{m}$, the series and parallel resonance of the mode shift apart, making $k_{\text {eff }}^{2}$ larger than $C_{m} / C_{o}$. As $Q$ increases, the two resonances move closer together, decreasing $k_{e f f}^{2}$, which approaches $C_{m} / C_{o}$ when $Q\left(C_{m} / C_{o}\right) \gg 1$ [6]. For the devices in this work, $Q\left(C_{m} / C_{o}\right) \sim$ $0.5-1$, a regime where the trade-off between $Q$ and $k_{\text {eff }}^{2}$ still exists. Consequently, the increase in $Q$ corresponding to increased cavity length $L$ leads to a drop in $k_{\text {eff }}^{2}$, as observed in simulation.

\section{FABRICATION}

Folded PnC resonators were fabricated in MIT's Microsystems Technology Lab using Raytheon's MMIC GaN-on-Si heterostructure, comprised of $\mathrm{AlGaN}(25 \mathrm{~nm}) / \mathrm{GaN}(1.7 \mathrm{um})$ grown on (111)-Si using Molecular Beam Epitaxy (MBE). A shallow AlGaN etch was used to remove the 2D electron gas (2DEG) between the Al$\mathrm{GaN} / \mathrm{GaN}$ layers and allow for transduction in the GaN layer. A 100 $\mathrm{nm}$ layer of $\mathrm{Ni}$ (used as the gate metal for GaN HEMTs) was then deposited and patterned to define piezoelectric IDTs. The choice of $\mathrm{Ni}$ for the electrodes is a departure from conventional Au electrodes found in GaN MMICs, as Au is mechanically lossy and is known to reduce resonator $Q$. Since these devices are processed side by side with GaN HEMTs, a PECVD $\mathrm{Si}_{3} \mathrm{~N}_{4}$ layer $(150 \mathrm{~nm})$ was deposited to passivate the surface and protect the 2DEG channel. A deep $\mathrm{Cl}_{2}$

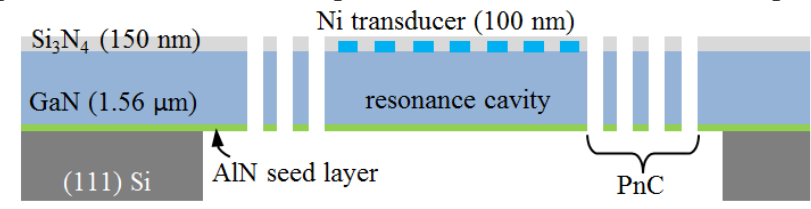

Figure 5: Cross section of MBE GaN resonator on $\mathrm{Si}$.

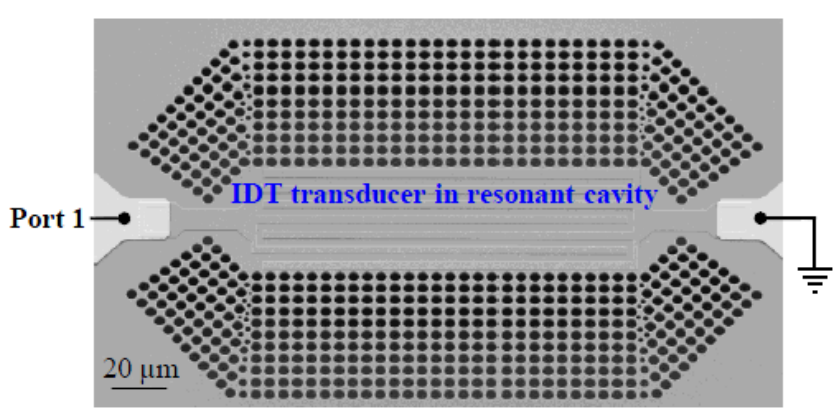

Figure 6: SEM of Device 1. IDT transducers (Ni) are routed into the resonant cavity through the openings. It is connected through vias to the probe pads, which are made of Au/Ti.

$\mathrm{GaN}$ etch then defined the PnCs and acoustic cavities. Metal pads $(50 \mathrm{~nm} \mathrm{Ti} / 300 \mathrm{~nm} \mathrm{Au})$ were then connected to the gate electrodes through vias in the passivation layer. Finally, a $\mathrm{XeF}_{2}$ etch released the resonators from the Si substrate. Fig. 5 depicts the cross section of the final suspended resonator. An SEM of one of the fabricated PnC resonators (Device 1 ) is shown in Fig. 6, with cavity length $L$ of $157 \mu \mathrm{m}$, folding angle $\theta=50^{\circ}$ and resonance at $516 \mathrm{MHz}$. This fabrication process is compatible with GaN HEMT technology [7].

\section{EXPERIMENTAL RESULTS AND ANALYSIS}

Devices were tested under vacuum in a Cascade PMC200 RF probe system. A standard 1-port S-parameter measurement was performed using an Agilent 5225A Network Analyzer with on-chip open de-embedding structure up to the routing electrodes outside the cavity. The measured 1-port device admittance was fitted to a modified Butterworth-Van Dyke circuit to extract resonance parameters, with a shunt capacitor and resistor used to model the feed-through.

We compare the performance of two devices with resonant dimension defined based on the structure in the simulation in Fig. 3 to demonstrate the behavior and design trade-offs of the folded PnC resonator. Labeled 'Device 1' and 'Device 2', the parameters of these resonators are provided in Table 1. Fig. 7 shows the wide-band and zoomed in (inset) frequency response of Device 1, exhibiting a clean spectrum over $>500 \mathrm{MHz}$ range.

Device 2 is identical apart from its cavity length, which is half that of Device 1. The 1-port frequency response of Device 2 is shown in Fig. 8. Reducing the cavity length by $2 \times$ decreases $Q$ by $2.5 \times$ but improves $k_{\text {eff }}^{2}$ by $2.3 \times$. This trend is in accordance with simulation (Fig. 3 ) in which a $4 \times$ decrease and $1.6 \times$ increase are expected for the same length scaling for $Q$ and $k_{\text {eff }}^{2}$, respectively.

Table 1: Measured performance comparison of GaN folded PnC resonators.

\begin{tabular}{|l|l|l|c|c|c|c|c|}
\hline & $\theta$ & $\begin{array}{c}a \\
(\mu \mathrm{m})\end{array}$ & $L / a$ & $\begin{array}{c}f_{o} \\
(\mathrm{MHz})\end{array}$ & $\begin{array}{c}R_{m} \\
(\mathrm{k} \Omega)\end{array}$ & $Q$ & $k_{\text {eff }}^{2}$ \\
\hline Dev. 1 & $50^{\circ}$ & 5.6 & 28 & 516 & 10 & 2924 & $0.10 \%$ \\
\hline Dev. 2 & $50^{\circ}$ & 5.6 & 14 & 528 & 30 & 1160 & $0.23 \%$ \\
\hline
\end{tabular}

In the interest of scaling to higher frequency, a third folded PnC resonator (Device 3) was demonstrated at $993 \mathrm{MHz}$, achieving an $f . Q$ product of $3.06 \times 10^{12}$. The PnC used for Device 3 has a band gap of from 940 to $1040 \mathrm{MHz}$. The SEM, frequency response, and fitted parameters for this structure are shown in Fig. 9. The harmonic spurious modes described by Eqn. 1 can be seen more clearly in Device 3, since the frequency difference between the fundamental mode and the first spurious mode is the same as in the case of Devices 1 and 2, but at $993 \mathrm{MHz}$ the fractional difference is smaller. 


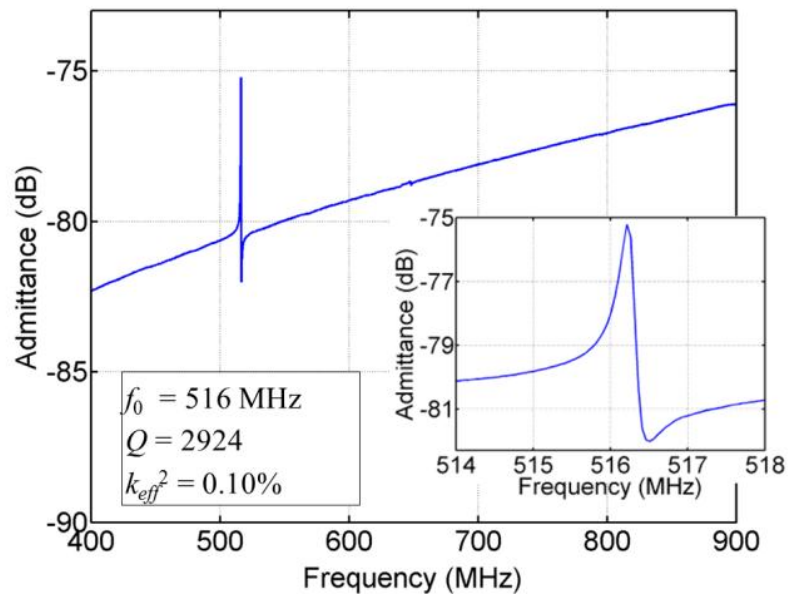

Figure 7: Measured frequency response of Device 1 with wide and narrow sweeps showing spurious-free spectrum.

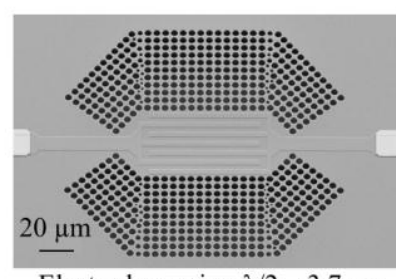

(a)

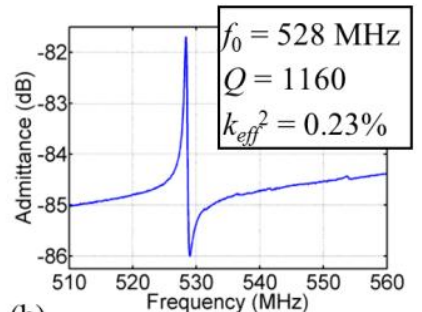

(b)

Figure 8: (a) SEM of Device 2, with L/a = 14. (b) Measured 1-port frequency response and fitted resonator parameters.

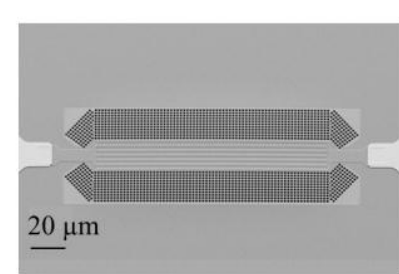

(a)

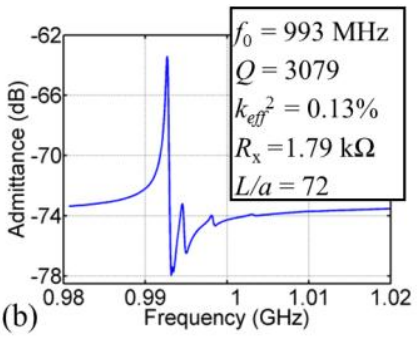

(b)

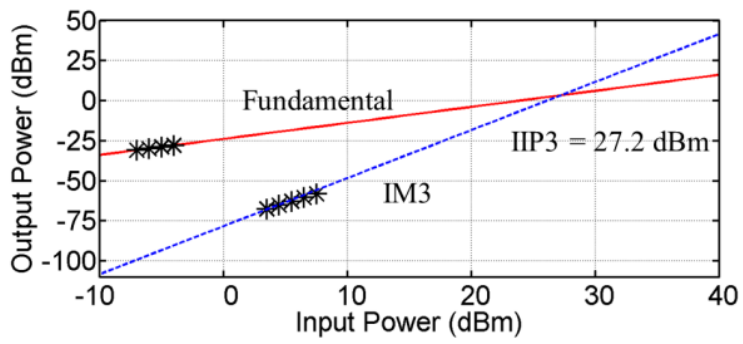

Figure 10: Power handling measurement of Device 3 at $993 \mathrm{MHz}$, using input tones spaced at $300 \mathrm{kHz}$ and $600 \mathrm{kHz}$. The IIP3 of $+27.2 \mathrm{dBm}$ is the highest reported in GaN resonators to date.

been calibrated to represent the power incident onto the device input port. The folded structure PnC resonator exhibits IIP3 of +27.2 $\mathrm{dBm}$, the highest reported in a $\mathrm{GaN}$ resonator to date. This is attributed to improved thermal conductance offered by the $\mathrm{PnC}$ boundaries relative to tethered structures.

\section{CONCLUSION}

We introduce a new RF MEMS resonator using a folded PnC structure to achieve wide band spurious mode suppression. Devices show clean frequency response over a wide bandwidth $(>500 \mathrm{MHz})$. At $993 \mathrm{MHz}$, high f. $Q$ product of $3.06 \times 10^{12}$ was demonstrated, on par with the best results in $\mathrm{GaN}$ to date. Furthermore, the device exhibited large power handling of over $+27 \mathrm{dBm}$ afforded by improved thermal dissipation in the large-perimeter PnC. This design leverages GaN MMIC technology, providing a low barrier-to-entry solution for monolithic timing and RF wireless communication applications including GHz MEMS front end band pass filters with excellent spurious mode suppression, linearity, and frequency selectivity.

\section{ACKNOWLEDGMENT}

The authors thank Brian Schultz and Thomas Kazior at Raytheon for GaN growth and process discussions, and Prof. Steven Johnson at MIT for helpful discussion. This work was funded by DRAPA DAHI Foundry N66001-13-1-4022 and NSF Career EECS-1150493.

\section{REFERENCES}

[1] M.F. Su, R. Olsson, Z. Leseman, El-Kady, "Realization of a phononic crystal operating at gigahertz frequencies," Applied Physics Letters, 96, 053111 (2010).

[2] N. Kuo, S. Gong, G. Piazza, "Ultra high frequency phononic crystal in silicon carbide," Solid-State Sensors, Actuators and Microsystems Conference (TRANSDUCERS'11), 2486-2489, (2011).

[3] S. Mohammadi, A. Adibi, et al., "High-Q micromechanical resonators in a two-dimensional phononic crystal slab," Applied Physics Letters, 94, 051906, (2009).

[4] S. Mohammadi, A. Adibi, "Waveguide-based phononic crystal micro/nanomechanical high-Q resonators," J. Microelectromechanical Systems, 21, 379-384, (2012).

[5] C.-Y. Huang, J.-H. Sun, T.-T. Wu, "A two-port ZnO/Silicon Lamb wave resonator using phononic crystals," Applied Physics Letters, 97, 031913, (2010).

[6] R. Ruby, et al., "After 60 years: A new formula for computing quality fact or is warranted," Ultrasonics 2008, pp. 431-436.

[7] L.C. Popa, D. Weinstein, "Switchable piezoelectric transduction in AlGaN/GaN MEMS resonators," Solid-State Sensors, Actuators and Microsystems (TRANSDUCERS'13),2461-64, (2013).

CONTACT Siping Wang; spinwang@ mit.edu 